\title{
Influence of a Cu-Nb Interface on Local Lattice Diffusivity in Cu During Irradiation
}

Shimin Mao ${ }^{1}$, Shen Dillon ${ }^{1,2}$ and Robert Averback ${ }^{1,2}$

1. Department of Materials Science and Engineering, University of Illinois Urbana Champaign, IL, 61801, USA

2. Frederick Seitz Materials Research Laboratory, University of Illinois Urbana Champaign, IL, 61801, USA

Radiation tolerant materials are essential to next-generation reactors that will provide safe, economical, and reliable energy. The generation and recombination of point defects underlies many of the damage and healing mechanisms that occur during irradiation. Promoting effective vacancy-interstitial recombination provides a general approach to suppressing radiation-induced damage. ${ }^{1,2}$ Interfaces, which serve as efficient sinks for point defects, have been intensively investigated for decades. Unfortunately, little is known experimentally about the local transport and spatial distribution of defects near interfacial sinks. Here $\mathrm{Cu}-\mathrm{Nb}$ interfaces are examined as a model interface between immiscible fcc and bcc alloys. The system has already been the subject of significant computational and experimental investigation due to its relative stability during irradiation. ${ }^{3-5}$

In this work, $\mathrm{Cu}-\mathrm{Nb}$ multilayers were grown by magnetron sputtering. A dilute chemical tracer $\mathrm{Cu}_{90} \mathrm{Au}_{10}$ was placed at different positions within the $\mathrm{Cu}$ matrix in order to probe the local diffusivity, which ultimately relates to the local vacancy concentration. The Au diffusion profiles after irradiation at different doses and temperatures were quantified by energy dispersive spectroscopy (EDS) in the STEM. Needle-shaped TEM samples, fabricated in the FIB, allow their thickness to be determined directly, aiding EDS quantification. Figure 1 shows an example image of the structures before and after irradiation. Radiation-enhanced diffusion (RED) of $\mathrm{Au}$ in $\mathrm{Cu}$ was measured by observing the broadening of the initial tracer layer. Figure 2 depicts the composition profiles of the Au before and after irradiation for tracers at different distances from the interface. This method allows us to spatially resolve diffusivity and vacancy concentration to distances of $\sim 25 \mathrm{~nm}$.

References:

[1] MJ Demkowicz, P Bellon, BD Wirth, MRS Bulletin 35 (2010), 992.

[2] R Sizmann, Journal of Nuclear Materials 69\&70 (1978), 386.

[3] MJ Demkowicz, RG Hoagland, JP Hirth, Physical Review Letters 100 (2008), 136102.

[4] X Zhang, N Li, O Anderoglu, H Wang, JG Swadner, T Hochbauer, A. Misra, RG Hoagland, Nuclear Instruments and Methods in Physics Research B 261 (2007), 1192.

[5] X Zhang, EF Fu, A Misra, MJ Demkowicz, JOM 62(12) (2010), 75.

[6] The authors acknowledge funding from the United States Department of Energy Basic Energy

Sciences under Grant DEFG02-05ER46217 
Figure 1a) Z-contrast STEM image of an as grown sample; b) and a similar sample after irradiation to $8 \times 10^{14} 1.8 \mathrm{MeV} \mathrm{Kr}^{+}$at $300^{\circ} \mathrm{C}$

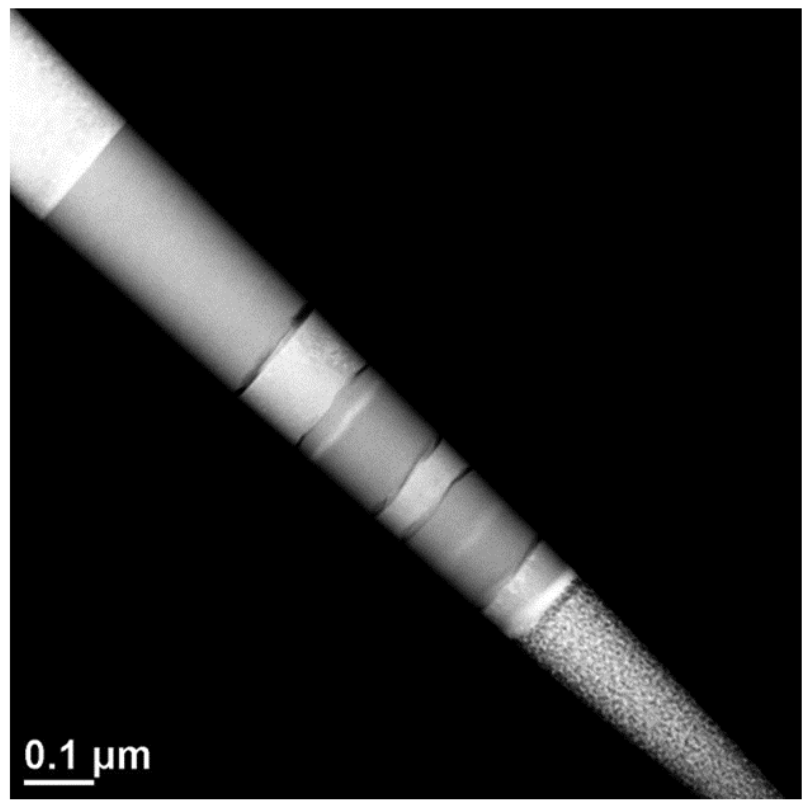

(a)

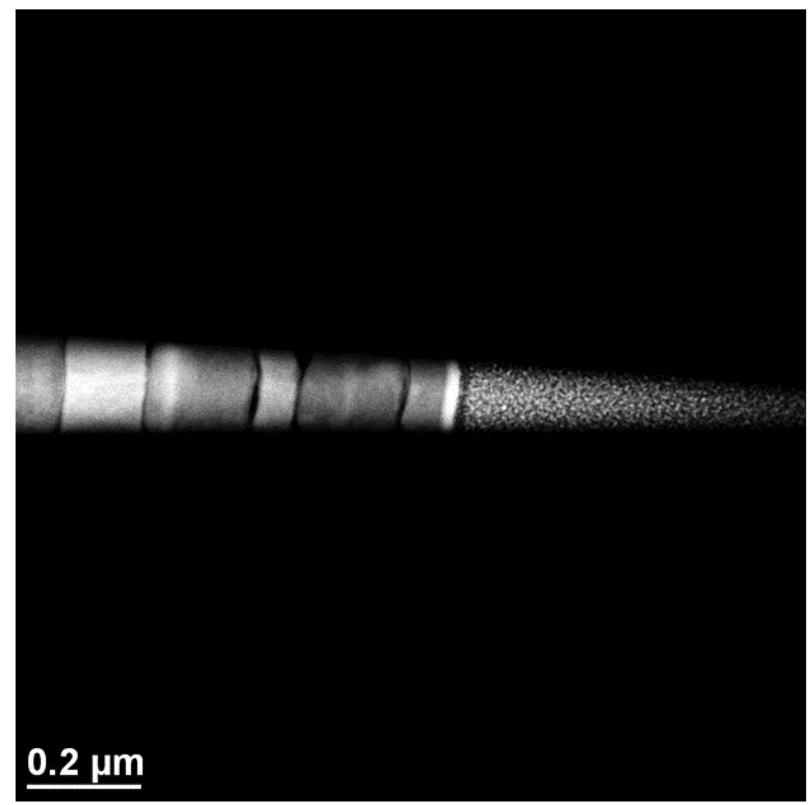

(b)

Figure 2 The Au chemical tracer diffusion profiles for the samples depicted in Figure 1 (black: as grown sample; red: irradiated).

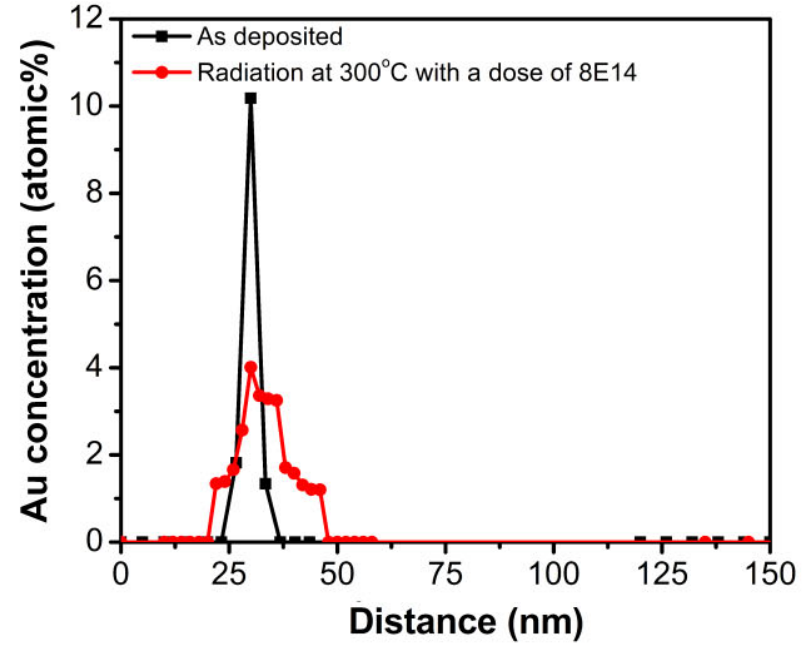

(a)

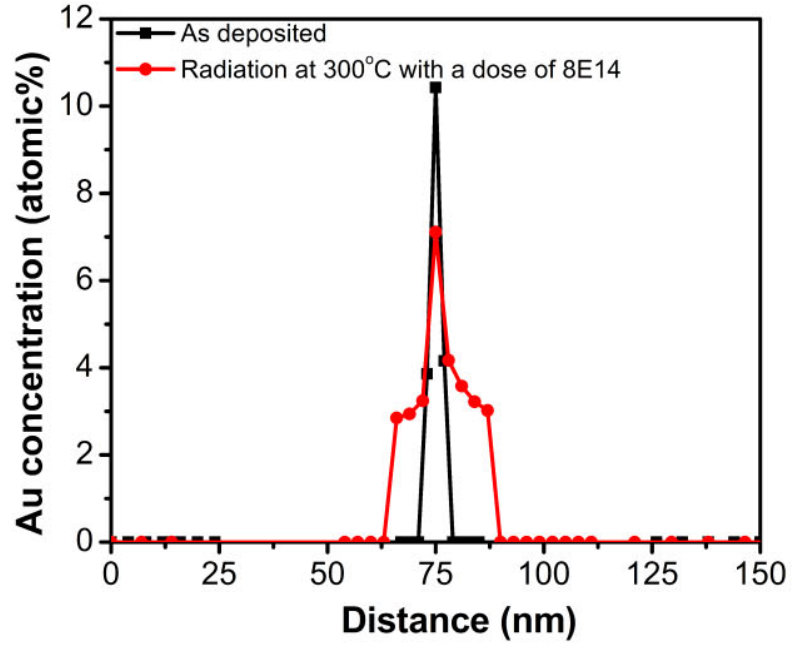

(b) 